\title{
Grid Enabled Molecular Dynamics: classical and quantum algorithms
}

\author{
S. C. Farantos ${ }^{a, b, 1}$, S. Stamatiadis ${ }^{a, b}$, L. Lathouwers ${ }^{c}$ and R. Guantes ${ }^{d}$ \\ ${ }^{a}$ Institute of Electronic Structure and Laser, Foundation for Research and Technology-Hellas, \\ Iraklion 711 10, Crete, Greece. \\ ${ }^{b}$ Department of Chemistry, University of Crete, Iraklion 711 10, Crete, Greece. \\ ${ }^{c}$ Department of Wiskunde-Informatica, University of Antwerp, Groenenborgerlaan 171, B2020, \\ Antwerp, Belgium. \\ ${ }^{d}$ Instituto de Matematicas y Fisica Fundamental, Consejo Superior de Investigaciones Cientificas, \\ Serrano 123, 28006 Madrid, Spain.
}

Received 5 August, 2005; accepted in revised form 12 August, 2005

\begin{abstract}
Molecular simulations have become a powerful tool in investigating the microscopic behavior of matter as well as in calculating macroscopic observable quantities. The predictive power and the accuracy of the methods used in molecular calculations are closely related to the current computer technology. Thus, the rapid advancement of Grid computing, i.e. the utilization of geographically distributed computers connected by relatively high latency networks, is expected to influence extensively the progress of computational sciences, provided algorithms which can utilize the hundreds and even thousands of the available computers in the Grid exist. In this lecture we review some of our computational methods, classical and quantum, used in small molecules, which seem promising for studying large scale in time and molecular size problems. In particular, in classical molecular simulations we are searching for specific trajectories connecting two regions of phase space (rare events) by solving two-point boundary value problems with multiple shooting techniques. In quantum dynamics we argue that using variable order finite difference methods for solving the Schrödinger equation in a cartesian coordinate system result in sparse Hamiltonian matrices which can make large scale problem solving feasible.
\end{abstract}

Keywords: Quantum molecular dynamics, classical molecular dynamics, finite differences, multiple shooting methods, Grid computing.

Mathematics Subject Classification: 0.2.60-x

PACS: $0.2 .60 . \mathrm{Lj}, 0.2 .70 . \mathrm{Bf}, 02.70 . \mathrm{Ns}, 34.30 .+\mathrm{h}, 82.20 . \mathrm{Ln}$

\section{Introduction}

Computational Chemistry is a topic whose progress is closely related to the current technology of computers. Molecular simulations are important in designing new materials, pharmaceuticals, in Biological Chemistry and Physics. In general, Molecular Computational Sciences face two challenges in the twenty first century. The first has to do with the size of the systems; studies which cover molecules from two atoms to nanostructures and the macroscopic states of matter are required. The second challenge is related to time and the dynamics of the systems; phenomena

\footnotetext{
${ }^{1}$ Corresponding author. E-mail: farantos@iesl.forth.gr
} 
which last from femtoseconds to seconds are important in material and biological sciences. Grid (distributed) computing could be the solution in bridging the gaps in time and space. A Grid of computers is envisioned as a seamless, integrated, computational and collaborative environment embracing different categories of distributed systems. Following the classification introduced by Foster and Kesselman ("The Grid: the blueprint for a new computing infrastructure") [1], computational Grids are categorized into five major classes of applications. In summary, these classes identify a specific context of applications such as supercomputing applications, high-throughput computing, on-demand computing, data-intensive computing and collaborative computing. This new paradigm in scientific computing is rapidly developing. Several Grid testbeds have been deployed around the world among which the European Grid. The ENACTS project [2] with its well planned and in depth studies has shown that Grids of computers are well developed not only at the national level but also as a European multi-national integration.

In our days, the most successful computational Grid model is the one based on internet by accessing thousands of PCs via running the programs as screensavers. The first such application was SETI@home to analyze the data from radio telescopes looking for signs of extraterrestrial life. Taking its inspiration from SETI, a protein folding project called Folding@Home (http://www.stanford.edu/group/pandegroup/folding) has been in operation at Stanford University for several years. Another active academic project is Predictor@home (http://predictor. scripps.edu/) which is aiming to structures prediction. This is a pilot of the Berkeley Open Infrastructure for Network Computing (BOINC), a software platform for distributed computing using volunteer computer resources. Another action on the human protein folding problem has been taken by the World Community Grid (WCG) (http://www.worldcommunitygrid.org/) which is supported by United Devices (UD). UD also runs the life-science research hub (http://www.grid.org/) to search for drug candidates to treat the smallpox virus.

In order to use successfully a worldwide distributed computing environment of hundreds or even thousands of heterogeneous processors such as the Grid, communications among these processors should be minimum. There are not many molecular simulations using the Grid environment such as to allow us to point out the strategy one should adopt in writing codes for molecular applications. A practical rule is to allow each processor to work independently, even though calculations are repeated, and only if something important happens to one of them, then they communicate. The common ingredient of the above successful applications in Internet Grid Computing is the computational algorithm employed, which guarantees minimum and asynchronous communications. Obviously, there are few problems which can be solved with such algorithms. Most of the interesting problems of Computational Chemistry require solutions of a large number of linear equations that can not be solved without significant communication among the computer nodes. On the other hand, parallelized codes written for large parallel machines may be of no good use when thousands of computers should be exploited connected by high latency networks. Thus, new algorithms and new programming paradigms suitable for distributed computing should be investigated in order to exploit Computational Grids [3].

In this lecture we review some of our methods used in classical and quantum dynamics of highly excited small molecules and we examine their potential to be utilized in a Grid computational environment. In quantum dynamics we shall investigate the combination of time evolution of wave packets in cartesian coordinate representations with angular momentum projections and the realization of this technique via generalizable time evolution schemes and variable order finite differencing methods for computing the derivatives. With respect to classical dynamics we shall explore the idea of searching specific trajectories which connect regions of phase space that correspond to different conformations of the molecule by formulating the problem as a two-point boundary value and using multiple shooting techniques. 


\section{Computational Methods}

Our understanding of basic molecular phenomena is strongly based on our ability to successfully solve the Schrödinger equation for realistic molecular systems. This is the aim of Quantum Molecular Dynamics (QMD) and it is the way to obtain reliable quantitative predictions. However, very useful physical insight can be gained from the use of a causal time dependent picture where, for instance, the path of a molecular encounter or a vibrational motion can be traced in configuration space. Therefore, a lot of efforts have been directed towards the development of methods which combine classical description with quantum corrections. Accurate wave packet propagation, together with knowledge of the proper classical orbits of the system, is one of the most complete views that we can have for a molecular process because exact numerical results are supplemented by a very clear physical picture. Such a program is accomplished by locating families of periodic orbits which then can be used to determine initial configurations for the wave packets $[4,5]$.

In the next two subsections we describe our approaches to QMD and Classical Molecular Dynamics (CMD) which are based on discretizing the configuration space and time respectively. The grid representation of space and time make the algorithms suitable for using them in a Grid of computers.

\subsection{Quantum Molecular Dynamics}

Grid methods for the solution of the Schrödinger equation are nowadays one of the most powerful and exploited tools both for the time independent (known as Discrete Variable Representation (DVR)) and time dependent pictures. Concerning the last one, it was the introduction of the Fourier Pseudospectral (PS) method which provided the necessary accuracy and computational efficiency to compete with the traditional variational techniques and to make feasible the task (for an excellent review see Ref.[6]). In the time dependent picture or wave packet propagation, the wave packet is advanced in time by an evolution operator which, if the Hamiltonian $\hat{H}$ is time independent, is an exponential function $\hat{U}(t ; \hat{H})=e^{-i \hat{H} t}$ (we put $\hbar=1$ ). This can be approximated by, for instance, a polynomial expansion [7]. The basic operation is therefore reduced to the evaluation of the action of the Hamiltonian operator onto the wave packet, $\hat{H} \Psi$. In long time propagation this operation has to be repeated many times, and thus, its efficiency will generally determine the computational cost of the problem. In practice, the wave function is often discretized in a grid using a collocation method and the Hamiltonian operator is represented by a matrix. If we considered only local operators this matrix would be diagonal, but the Hamiltonian includes the kinetic energy operator which is non local in the coordinate domain. If the number of collocation points in each coordinate $\alpha$ is $n_{\alpha}$, the Hamiltonian matrix will contain of the order of $N \times n$ nonzero elements, where $N$ is the total number of collocation points $N=\prod_{\alpha} n_{\alpha}$ and $n=\sum_{\alpha} n_{\alpha}$.

In the time independent picture one is concerned with the diagonalization of the Hamiltonian matrix constructed in the same way to obtain the eigenvalues and eigenvectors. Usually, an iterative procedure such as the Lanczos method and variants [8] is used for efficient diagonalization, which again involves products of the Hamiltonian matrix with vectors as the basic step. It is clear that important computational savings are possible when the Hamiltonian matrix is sparse or its action on the wave function can be calculated efficiently.

A way to increase significantly the sparsity of the Hamiltonian matrix is by the use of local methods. Here, local means that the action of the kinetic energy operator (or the Laplacian) on the wave function is approximated by using only local information or neighboring grid points. Although the derivative of a function is a local property, the wave function is defined on the whole configuration space and a piecewise representation of a function by a local polynomial approximation, generally, converges more slowly than a spectral representation.

In this work we review some recent advances in Finite Difference algorithms which may result 
in sparse Hamiltonian matrices. However, developing algorithms for QMD suitable to use the Grid computing technologies we must first decide the coordinate system to express the molecular Hamiltonian.

\subsubsection{The angular momentum projection method}

In their 1996 review of Quantum Molecular Dynamics [9] R.E. Wyatt and J.Z.H. Zang state "it is very important to develop and apply methods that can be extended to moderately sized polyatomic molecules (5 to 12 atoms)". In spite of the diversity of theoretical approaches and the ever growing computational facilities it is safe to say that the calculation of vibration-rotation spectra or state to state cross sections for five or more atomic systems is all but routine. We will first examine the reasons for this state of affairs and then propose a scheme that can satisfy the above ambitions.

The standard way of describing a polyatomic system in the absence of external fields is to introduce the center of mass, three Euler angles and $3 N-6$ internal coordinates. The Hamiltonian in mass weighted cartesian coordinates $x_{k m}$ is written

$$
\hat{H}=-\frac{1}{2} \sum_{k, m} \frac{\partial^{2}}{\partial x_{k m}^{2}}+V(R),
$$

where, $k=1,2,3$ denotes the three cartesian coordinates, $m=1, \ldots, N$ the atoms and $V(R)$ the potential energy as a function of the internal coordinates $R$.

The transformation of the original cartesian Hamiltonian to the above general curvilinear coordinates yields the generic form

$$
\hat{H}=\hat{H}_{0}\left(q, \frac{\partial}{\partial q}\right)+\sum_{k} A_{k}\left(q, \frac{\partial}{\partial q}\right) J_{k}+\sum_{k, l} \frac{1}{2} I_{k l}^{-1}(q) J_{k} J_{l} .
$$

$J_{k}$ denote the components of angular momentum and $I_{k l}$ are the components of the moment of inertial tensor.

In the above equation we recognize the vibrational, rotational and the vibrational-rotational coupling parts of the Hamiltonian. We will refer to the derivation of Eq. (2) as frame transformation (FT). The problem with Eq. (2) is twofold. First, there is the analytical derivation of the Hamiltonian parts in curvilinear coordinates $q_{1}, \ldots, q_{3 N-6}$, and Euler angles $\phi, \theta, \gamma$ or angular momentum operators. Not only this is a cumbersome task for more than three atoms but this procedure has to be repeated for every choice of curvilinear coordinates and angles. Secondly, the general expression for the vibrational Hamiltonian reads

$$
\hat{H}_{0}\left(q, \frac{\partial}{\partial q}\right)=-\frac{1}{2} \sum_{i, j} \frac{\partial}{\partial q_{i}}\left(\sqrt{g} g^{i j} \frac{\partial}{\partial q_{j}}\right)+V(q),
$$

where $g^{i j}$ is the metric tensor of the curvilinear coordinates. The appearance of cross derivatives implies that the number of partial differential operators necessarily exceeds $(3 N-6)(3 N-5) / 2$. Therefore, the computational effort scales as $N^{2}$.

The question then arises whether an alternative theoretical approach can be devised that avoids the inherent problems of frame transformation and that can readily be extended to five or more atoms. Our basic starting point is to not separate rotations at the operator level but to use projection techniques to conserve total angular momentum. An alternative version of Eq. (2) is to introduce $N$ Jacobi vectors the last one of which is the center of mass. The Hamiltonian can then be written as

$$
\hat{H}=-\frac{1}{2} \sum_{k, m} \frac{\partial^{2}}{\partial u_{k m}^{2}}+V(R(u))
$$


which contains the $3 N-3$ kinetic energies of the Jacobi coordinates $u_{k m}, k=1,2,3$ and $m=$ $1, \ldots, N-1$.

We first observe that all molecular properties such as spectra, cross sections, etc., can be obtained by Fourier transforming the appropriate time-correlation function. For specified total angular momentum, $J$, the vibration-rotation spectra require the calculation of the autocorrelation function

$$
C_{J}(t)=<\Psi(0)\left|\exp \left(-i \hat{H}_{J} t\right)\right| \Psi(0)>,
$$

while for other properties the appropriate operator has to be inserted in the matrix element. Consider the rotations about the center of mass which constitute the rotation group of the system. The corresponding operators on the system wave functions are

$$
R(\Omega)=\exp \left(-i \phi J_{z}\right) \exp \left(-i \theta J_{y}\right) \exp \left(-i \gamma J_{z}\right),
$$

while, the associated irreducible representations are the Wigner functions

$$
D_{M K}^{J}(\Omega)=\exp (-i M \phi) d_{M K}^{J}(\theta) \exp (-i K \gamma) .
$$

From the group elements and the irreducible representations one can construct the group projection operators

$$
P_{M K}^{J}=\frac{2 J+1}{8 \pi^{2}} \int d \Omega D_{M K}^{J}(\Omega) R(\Omega) .
$$

Their properties have been listed and proven elsewhere [10]. The ones we need in the present context are

$$
\sum_{J} P_{J}=1, \text { with } P_{J}=\sum_{K} P_{K K}^{J},
$$

i.e., one can construct the projector onto the space of total angular momentum $J$ as the $K$ sum of the operators $P_{K K}^{J}$. The operators $P_{J}$, being true projection operators, are idempotent and hermitian and, because the Hamiltonian is rotationally invariant, they commute with $\hat{H}$

$$
P_{J}^{2}=P_{J}, \quad P_{J}^{\dagger}=P_{J}, \quad\left[\hat{H}, P_{J}\right]=0 .
$$

Since, the operator $\hat{H}_{J}$ appearing in Eq.(5) is really $P_{J} \hat{H} P_{J}$, one can rewrite the formula for the autocorrelation function as

$$
C_{J}(t)=<\Psi(0)\left|P_{J} \exp (-i \hat{H} t) P_{J}\right| \Psi(0)>.
$$

This expression is the key to the method. Indeed, it shows that evaluating $C_{J}(t)$ can be regarded as a three stage process: the projection of the initial state onto the subspace $J$, the time evolution of the initial state under the total Hamiltonian and the calculation of the overlap of the projected and time evolved states.

The projection of the initial state is a one time numerical integration and is computationally irrelevant versus the time evolutions. The time evolution of the $3 N-6$ variables involves cross derivatives in a $2 J+1$ dimensional matrix. The alternative propagation under Eq. (4) uses $3 N-3$ strictly separated variables. One can make a rough estimate of the ratio of the computational effort of calculating the action of Eq. (2) versus Eq. (4). Assuming that all variables are mapped on a grid of $P$ points we find $(2 J+1)(3 N-6) P^{-3}$.

However there is an extra savings device for the evolution under Eq. (4). Indeed, the wave function will not extend over the entire Jacobi hypercube but rather it is confined to the corresponding hypersphere. 


\subsubsection{Variable order finite difference method}

Now we proceed to compute the action of the Hamiltonian operator onto the wave function. In previous papers $[11,12]$, we reported results from the application of a variable order Finite Difference (FD) method to approximate the action of a Hamiltonian operator on the wave function in the time-dependent Schrödinger equation or the Hamiltonian matrix elements in the timeindependent picture. One, two and three dimensional model potentials in radial and angular coordinates were used to investigate the accuracy and the stability of these methods, whereas in a companion paper [13], the time-dependent Schrödinger equation was solved for the van der Waals system $\mathrm{Ar}_{3}$. The impetus for this project was given by recent advances in high order Finite Difference approximations. We mainly refer to the limit of infinite order Finite Difference formulas with respect to global Pseudospectral methods (PS) investigated by Fornberg [14], and Boyd's work [15] which views Finite Difference methods as a certain sum acceleration of Pseudospectral techniques.

Finite Difference approximations of the derivatives of a function $F(x)$ can be extracted by interpolating $F(x)$ with Lagrange polynomials, $P(x)$. This allows one to calculate the derivatives analytically at arbitrarily chosen grid points and with a variable order of approximation. The Lagrange fundamental polynomials of order $N-1$ are defined by

$$
L_{k}(x)=\prod_{j=1}^{N^{\prime}}\left(x-x_{j}\right) / \prod_{j=1}^{N^{\prime}}\left(x_{k}-x_{j}\right), \quad k=1,2, \ldots, N,
$$

where the prime means that the term $j=k$ is not included in the products. The values of $L_{k}\left(x_{j}\right)$ are zero for $j \neq k$ and one for $j=k$ by construction. The function can then be approximated as

$$
F(x) \approx P_{N}(x)=\sum_{k=1}^{N} F\left(x_{k}\right) L_{k}(x) .
$$

$P_{N}$ is a polynomial of order $N-1$. In Ref.[11] we discussed how FD is related to the Sinc-DVR method by taking the limit in the two above mentioned senses:

i) An infinite order limit of centered FD formulas on an equi-spaced grid yields the Discrete Variable Representation result when we use as a basis set the Sinc functions $(\operatorname{Sinc}(x) \equiv \sin (\pi x) / \pi x)$ $[14,17]$. Although, this limit is defined formally as $N$, the number of grid points used in the approximation, tends to infinity, some theoretical considerations [14] as well as numerical results [11] lead us to expect that the accuracy of the FD approximation is the same to that of the DVR method as we approach the full grid to calculate the FD coefficients.

ii) FD can also be viewed as a sum acceleration method which improves the convergence of the Pseudospectral approximation [15]. The rate of convergence is, however, non-uniform in the wave number, giving very high accuracy for low wave numbers and poor accuracy for wave numbers near the aliasing limit [18]. However, this does not cause a severe practical limitation, since, by increasing the number of grid points in the appropriate region we can have an accurate enough representation of the true spectrum in the range of interest. This is one property which makes FD useful as an alternative to the common DVR [19] and other PS methods such as Fast Fourier Transform techniques (FFT) $[6,7]$.

The current interest in Finite Difference methods is fully justified when solutions of the Schrödinger equation are required for multidimensional systems such as polyatomic molecules. The present most popular methods employed in quantum molecular dynamics are the Fast Fourier Transform and the Discrete Variable Representation techniques. FFT generally uses hypercubic grid domains which result in wasted configuration space sampling. A large number of the selected configuration points correspond to high potential energy values, which do not contribute to the eigenstates that 
we are seeking. Global DVR methods allow us to choose easily the configuration points which are relevant to the states we want to calculate, but still, we must employ in each dimension all grid points. Local methods such as FD have the advantages of DVR but also produce sparse matrices which make them more economical in computer memory and time provided the PS accuracy is achieved at lower order than the high order limit.

There are some other benefits for FD with respect to global Pseudospectral methods. Convergence can be examined not only by increasing the number of grid points but also by varying in a systematic way the order of approximation of the derivatives. Finite Difference methods may incorporate several boundary conditions and choose the grid points without necessarily relying on specific basis functions. The topography of the multidimensional molecular potential functions is usually complex. The ability of using non equi-spaced grids is as important as keeping the grid points in accordance to the chosen energy interval. The computer codes for a FD representation of the Hamiltonian can be efficiently parallelized, since the basic operation is the multiplication of a vector by a sparse matrix. The ongoing, active research in the relevant field produced various approaches to the efficient distribution of a sparse matrix, especially a "regular" one, i.e., one formed through the discretization of a differential equation on a grid, and the subsequent multiplication with an appropriately distributed vector. The developed methods tackle, in various degrees, the requirements of load balancing and minimal communication cost across a processor grid. The overview presented in [20] is a starting point for the available techniques.

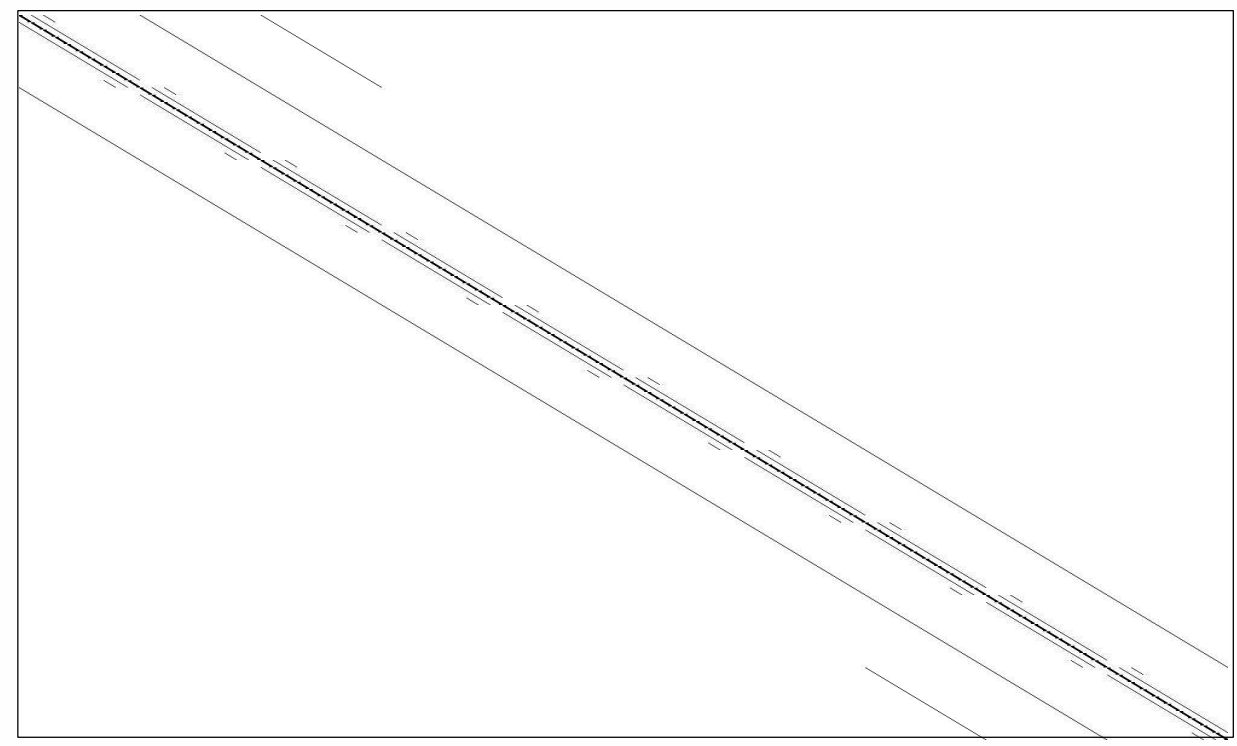

Figure 1: Pattern of a Hamiltonian matrix for a system of three degrees of freedom with the kinetic energy operator evaluated by finite difference approximation using three point stencils.

In Figure 1 we show schematically the Hamiltonian matrix when we employ a 3-point difference 
method to calculate the second derivatives in a three dimensional system. The high sparsity of the matrix is inherent, and this is the best for distributed computing.

As an example we will study a two dimensional system employed in several investigations in the past, mainly in the connection between classical and quantum dynamics. One of us [21] carried out extensive studies of the periodic orbit structure of this system and its relation to quantum mechanics. The system is described by the Hamiltonian:

$$
H=\frac{1}{2}\left(p_{x}^{2}+p_{y}^{2}\right)+\frac{1}{2}\left(\omega_{x}^{2} x^{2}+\omega_{y}^{2} y^{2}\right)-\epsilon x^{2} y,
$$

where the parameters are $\omega_{x}^{2}=0.9, \omega_{y}^{2}=1.6$ and $\epsilon=0.08$. The time dependent Schrödinger equation was solved numerically using a Chebychev expansion [7] for the propagation in time, while the action of the Hamiltonian operator on the wave function was evaluated with the FFT method and with matrix-vector multiplication using FD in order to study the convergence and compare the computation times. The resulting vibrational spectrum was obtained as usual with the Fourier transform of the autocorrelation function of the wave packet

$$
I(E)=\frac{1}{2 \pi} \int_{-\infty}^{\infty} \exp (i E t)<\Psi(\vec{x}, 0) \mid \Psi(\vec{x}, t)>d t .
$$

We propagated a Gaussian wave packet initially localized on a 1:2 resonance periodic orbit at a high energy ( $E=23$ a.u., see Fig. 12b in Ref.[21]). It is interesting to plot the wave packet in configuration space and compare the solution of the Schrödinger equation using the two methods. In both cases we used a rectangular grid with 64 points in each dimension, and $\Delta x=0.3175$. We wanted to obtain a high resolution for the spectrum $(\Delta E \simeq 0.056)$, and thus, we propagated the wave packet for 1024 time steps. At $t=1 / 4$ of the total time we took a snapshot of the wave function. In Fig. 2a we show the wave function obtained with the FFT method (the potential energy contour is superimposed on the same plot at the mean energy of the wave packet). In Figs $2 \mathrm{~b}, \mathrm{c}$ and $\mathrm{d}$ we show the same wave packet obtained with a second, fourth and seventh order Finite Difference approximation, respectively. The order of approximation $(M)$ yields stencils with $2 M+1$ points. From Fig. 2 we can see that convergence of the wave function is approached with the FD method, even for a wave packet quite spread in configuration space. Finer details reproduced by even higher order FD approximations, however, will not affect very much the spectrum, since, this is an average of the propagated wave function over the configuration space.

The resulting spectra are shown in Fig. 3 (here we omit the second order FD for the sake of clarity, although the comparison is very poor as expected). Even with order $M=7$, the only appreciable difference is in the intensities at high energies.

We recall that, a FD approximation is equivalent to a Taylor expansion series of the kinetic energy spectrum, therefore, in the limit of $\Delta x \rightarrow 0$ we recover the exact spectrum irrespectively of the order of the approximation. It is worth to investigate then how the FD converges to the Fourier method as we increase the order as well as we decrease the grid spacing. We examine the differences of the central eigenvalue $(E=22.18)$ from that obtained with the FFT method with 64 points in each dimension. When this difference is less than the resolution in the power spectrum the results are considered identical. Of course, for higher eigenvalues we should increase further the order or decrease the grid spacing to converge to the desired resolution, but the general behavior is seen in Fig. 4 where we used $64,80,100$ and 120 points $(\Delta x=0.3175,0.2532,0.2020$ and 0.1681 , respectively). It is seen that convergence in both directions is quite fast, although it is computationally cheaper on a single computer to increase the order of the approximation and take less grid points than to increase the number of grid points (by increasing the order by one we should add two more grid points to evaluate the Laplacian, but we should increase $N$ by about 20 points at low order to get the same reduction in the error). 

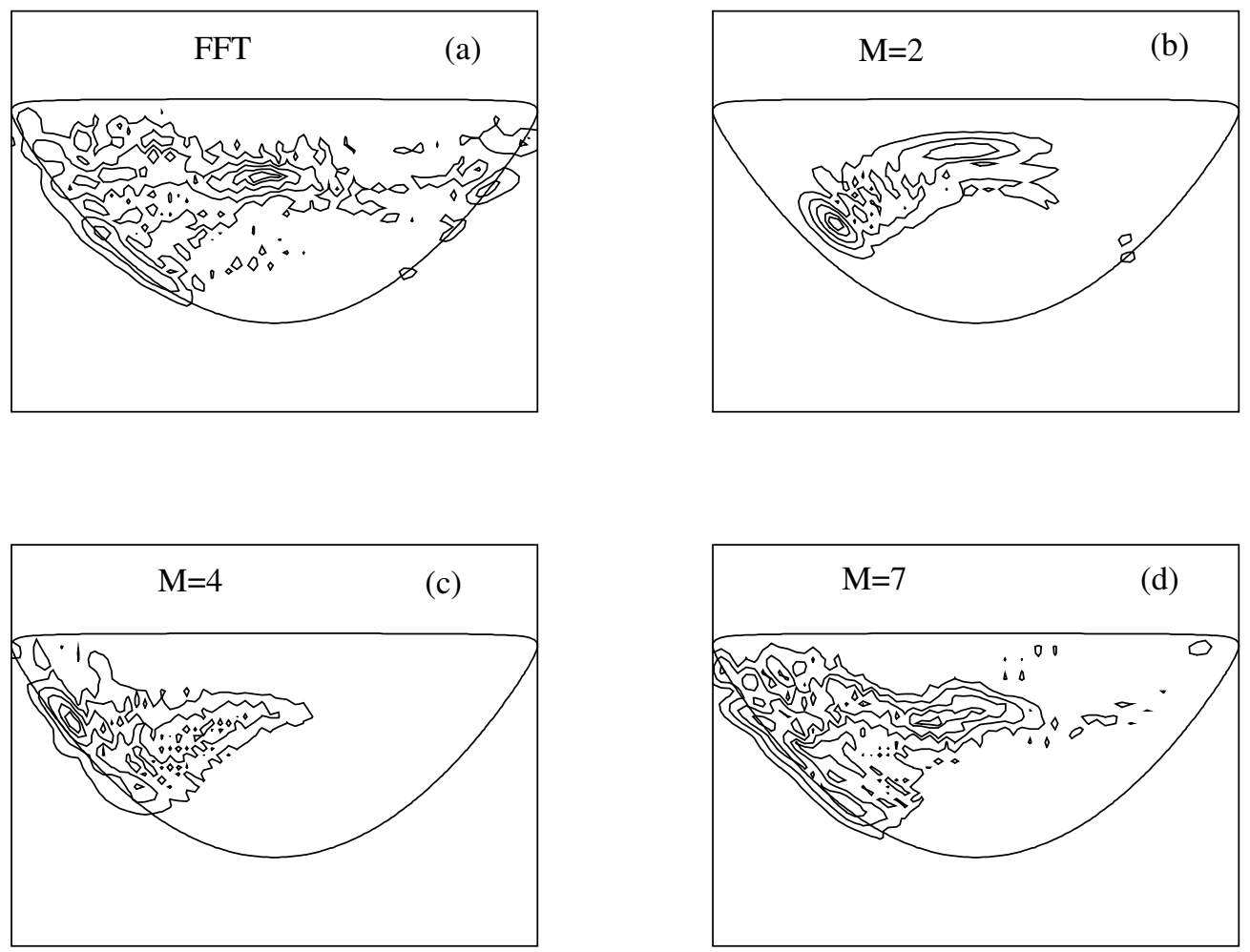

Figure 2: Snapshots of the wave packet at $t=28$ a.u. (1/4 of total propagation time) with a rectangular grid and 64 grid points in each dimension. (a) FFT method; (b) FD with $M=2$ (second order); (c) FD $4^{\text {th }}$ order; (d) FD $7^{\text {th }}$ order. The stencils are with $2 M+1$ points.

\subsection{Classical Molecular Dynamics}

The term Molecular Dynamics usually implies a Monte Carlo method which involves the integration of classical trajectories in phase space and a random selection for their initial conditions. Dealing with large molecules convergence is not an easy task and it usually requires proper sampling of phase space as well as the integration of the equations of motion for very long times or the sampling of a large number of trajectories. Therefore, it is useful if we know the type of trajectories we want to sample, for example those which visit the regions in configuration space of reactants and products in chemical reactions or trajectories which originate and end to particular phase space structures like stable and unstable manifolds. In those cases where the initial and final states of a trajectory satisfy a known relation, it is preferable to solve a two-point boundary value (2PBV) problem than randomly sampling initial conditions for integrating the equations of motion and accepting or rejecting trajectories according to the final state of the trajectory. A typical $2 \mathrm{PBV}$ problem is the location of periodic orbits (PO).

\subsubsection{The $2 P B V$ problem}

A $2 \mathrm{PBV}$ problem is formulated in the following way. The phase space of a molecule is described with the generalized coordinates $q_{i}(t), i=1, \ldots, N$ and their conjugate momenta $p_{i}(t), i=1, \ldots, N$ which are functions of time $t$. For the simplification of the mathematical equations we define the 


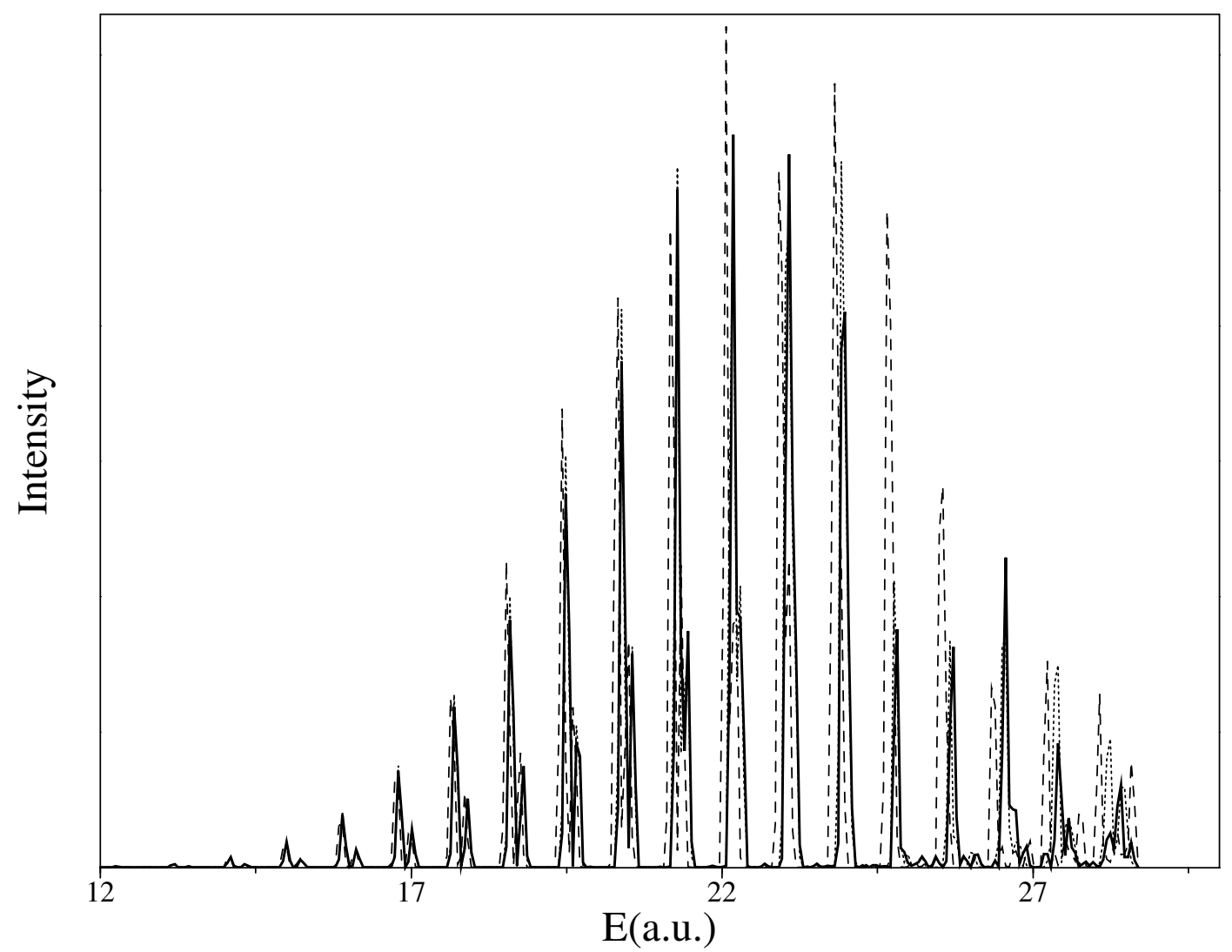

Figure 3: Power spectra obtained from the correlation function of the wave packet shown in Fig. 2. FFT(solid line), FD $4^{\text {th }}$ order (dashed line) and FD $7^{\text {th }}$ order (dotted line). The stencils are with $2 M+1$ points.

column vector

$$
\vec{x}=(\vec{q}, \vec{p})^{T} .
$$

Using $\vec{x}$ we can write Hamilton equations in the form

$$
\frac{d \vec{x}(t)}{d t}=\overrightarrow{\dot{x}}(t)=J \nabla H[\vec{x}(t)] \quad(0 \leq t \leq T)
$$

where $H$ is the Hamiltonian function, and $J$ is the symplectic matrix

$$
J=\left(\begin{array}{rr}
0_{N} & I_{N} \\
-I_{N} & 0_{N}
\end{array}\right)
$$

$0_{N}$ and $I_{N}$ are the zero and unit $N \times N$ matrices respectively. $J \nabla H(\vec{x})$ is a vector field, and $J$ satisfies the relations,

$$
J^{-1}=-J \text { and } J^{2}=-I_{2 N} .
$$

We want to find trajectories whose the initial, $\vec{x}(0)$, and final point, $\vec{x}(T)$, satisfy the equation

$$
\vec{B}[\vec{x}(0), \vec{x}(T) ; T]=0 \text {. }
$$




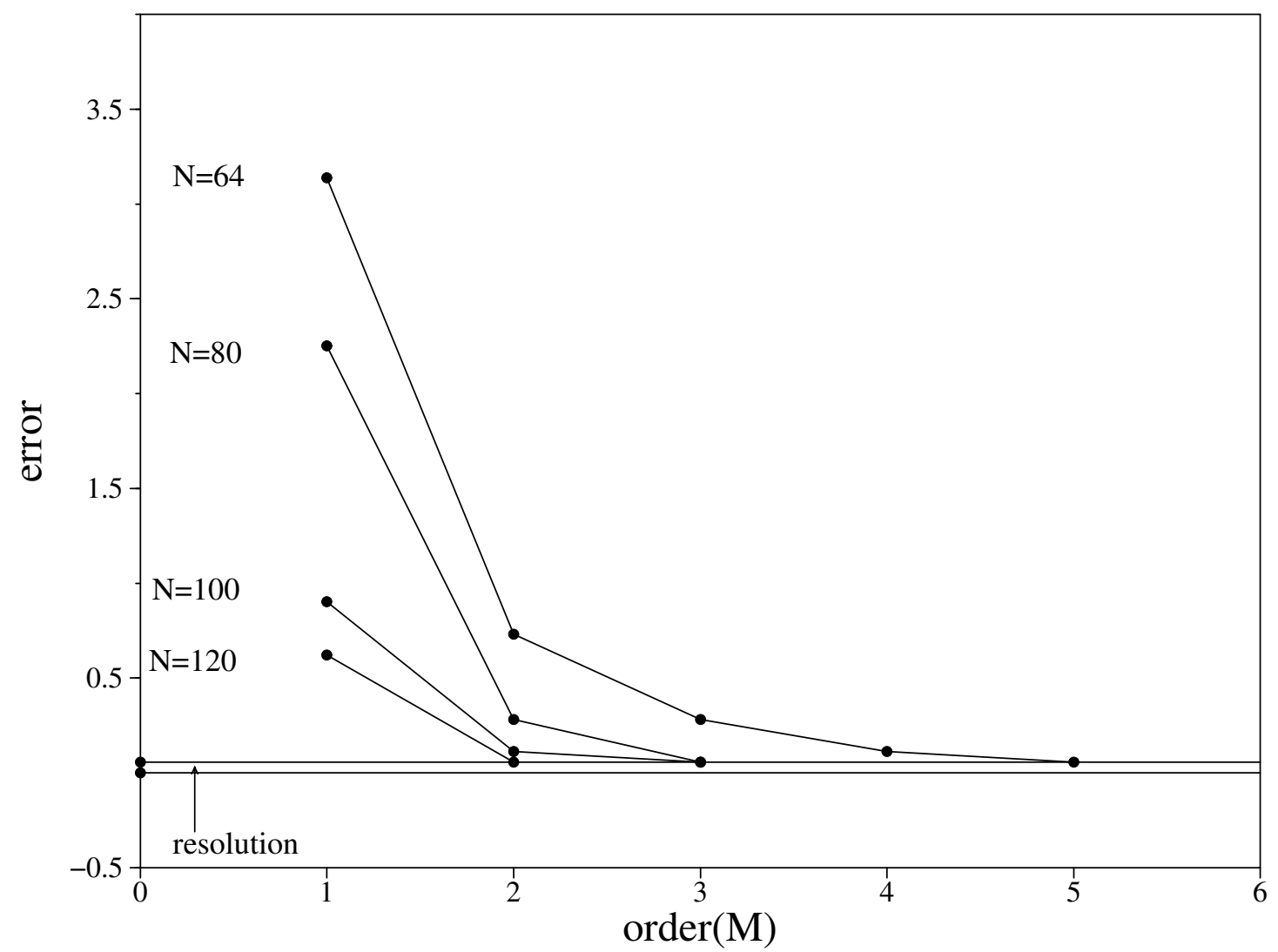

Figure 4: Differences in energy with respect to the central eigenvalue $(E=22.18)$ in the FFT spectrum of Fig. 3 as a function of the order of approximation. Rectangular grids with 64, 80, 100 and 120 points were used.

Treating the time $T$ as a parameter we are searching for families of trajectories which are solutions of the Hamilton equations of motion and simultaneously satisfy the two-point boundary conditions, Eq. (20). For example, trajectories with known distance of the initial and final points

$$
\vec{x}(T)-\vec{x}(0)=\vec{\xi}
$$

the boundary conditions are

$$
\vec{B}[\vec{x}(0), \vec{x}(T)]=\vec{x}(T)-\vec{x}(0)-\vec{\xi}=0 .
$$

$\xi=0$ yields the boundary conditions for periodic orbits.

The common procedure to solve a $2 \mathrm{PBV}$ problem is to cast it into an initial value problem through an iterative method (Ref.[5] and references therein). We consider the initial values of the coordinates and momenta, $\vec{s}$, of an approximate solution

$$
\vec{s}=\vec{x}(0),
$$

as independent variables of the nonlinear functions

$$
\vec{B}(\vec{s} ; T)=\vec{B}[\vec{s}, \vec{x}(T ; \vec{s})]
$$


$\vec{B}$ parametrically depends on the period $T$. We denote the roots of boundary equations as $\vec{s}_{*}$, i.e.,

$$
\vec{B}\left(\vec{s}_{*} ; T\right)=0 .
$$

Hence, if $\vec{s}$ is a nearby value to the solution $\vec{s}_{*}$ we can compute the functions $\vec{B}(\vec{s})$ by integrating Hamilton equations for the time interval $[0, T]$. By appropriately modifying the initial values $\vec{s}$ we hope to converge to the solution, that is, $\vec{s} \rightarrow \vec{s}_{*}$ and $B \rightarrow 0$.

A common procedure to find the roots of Eqs (25) is the Newton-Raphson method. This is an iterative scheme and at each iteration, $k$, we update the initial conditions of the orbit

$$
\vec{s}_{k+1}=\vec{s}_{k}+\Delta \vec{s}_{k} .
$$

The corrections $\Delta \vec{s}_{k}$ are obtained by expanding Eqs (25) in a Taylor series up to the first order

$$
\vec{B}\left(\vec{s}_{k+1} ; T\right)=\vec{B}\left(\vec{s}_{k}+\Delta \vec{s}_{k} ; T\right) \approx \vec{B}\left(\vec{s}_{k} ; T\right)+\frac{\partial \vec{B}}{\partial \vec{s}_{k}} \Delta \vec{s}_{k}=0,
$$

or

$$
\vec{B}\left(\vec{s}_{k} ; T\right)+\left[\frac{\partial \vec{B}}{\partial \vec{s}_{k}} Z_{k}(0)+\frac{\partial \vec{B}}{\partial \vec{x}_{k}(T)} Z_{k}(T)\right] \Delta \vec{s}_{k}=0 .
$$

The matrix (Jacobian)

$$
Z_{k}(t)=\frac{\partial \vec{x}_{k}\left(t ; \vec{s}_{k}\right)}{\partial \vec{s}_{k}}
$$

is the Fundamental Matrix, which is evaluated by integrating the variational equations

$$
\overrightarrow{\dot{\zeta}}(t)=A(t) \vec{\zeta}(t) \quad(0 \leq t \leq T),
$$

where the second derivatives of the Hamiltonian with respect to coordinates and momenta are needed

$$
A(t)=J \partial^{2} H[\vec{x}(t)] .
$$

These equations calculate the linearized part of the difference of two initially neighboring trajectories in time, $\vec{\zeta}=\vec{x}^{\prime}-\vec{x}$. The Fundamental Matrix is also a solution of the variational equations as can be seen by taking the time derivatives of Eq. (29)

$$
\dot{Z}(t)=A(t) Z(t) .
$$

Thus, to perform the $k^{t h}$ iteration in the Newton-Raphson method we first integrate for time $[0, T]$ the differential equations

$$
\begin{aligned}
\overrightarrow{\dot{x}}_{k}(t) & =J \nabla H\left[\vec{x}_{k}(t)\right] \\
\dot{Z}_{k}(t) & =A_{k}(t) Z_{k}(t),
\end{aligned}
$$

with initial conditions

$$
\begin{aligned}
& \vec{x}_{k}(0)=\vec{s}_{k} \\
& Z_{k}(0)=I_{2 N} .
\end{aligned}
$$

Then, we solve the linear algebraic equations

$$
\left[\frac{\partial \vec{B}}{\partial \vec{s}_{k}} Z_{k}(0)+\frac{\partial \vec{B}}{\partial \vec{x}_{k}(T)} Z_{k}(T)\right] \Delta \vec{s}_{k}=-\vec{B}\left(\vec{s}_{k} ; T\right),
$$

in order to find the initial conditions for the $(k+1)^{t h}$ iteration (Eq. (26)). 
For a periodic orbit the boundary conditions become

$$
\left[Z_{k}(T)-I_{2 N}\right] \Delta \vec{s}_{k}=-\vec{B}\left(\vec{s}_{k} ; T\right) .
$$

The Fundamental Matrix at period $T$ is called Monodromy Matrix, $M=Z(T)$, and its eigenvalues are used for the stability analysis of the periodic orbit. Details can be found in Ref.[22].

Once we have located one member of the family of the trajectories that we are looking for, we can apply continuation techniques [23] to find trajectories at different times $T$. This is done by using $T$ as the control parameter. Usually, for small increments of the time $T$ linear extrapolation methods are sufficient [5].

\subsubsection{The multiple node $2 P B V$ algorithm}

Let us assume that we divide the time $T$ in $(m-1)$ time intervals by choosing $m$ nodes. The idea of the multiple shooting method is to integrate $m-1$ trajectories -one for each time sub-intervaland to check if the final values of each trajectory coincide with the initial conditions of the next. To formulate this idea we introduce the scaled time, $\tau=t / T, \quad(0 \leq \tau \leq 1)$,

$$
0=\tau_{1}<\tau_{2}<\cdots<\tau_{m-1}<\tau_{m}=1 .
$$

Thus, for the simple shooting method $m=2$.

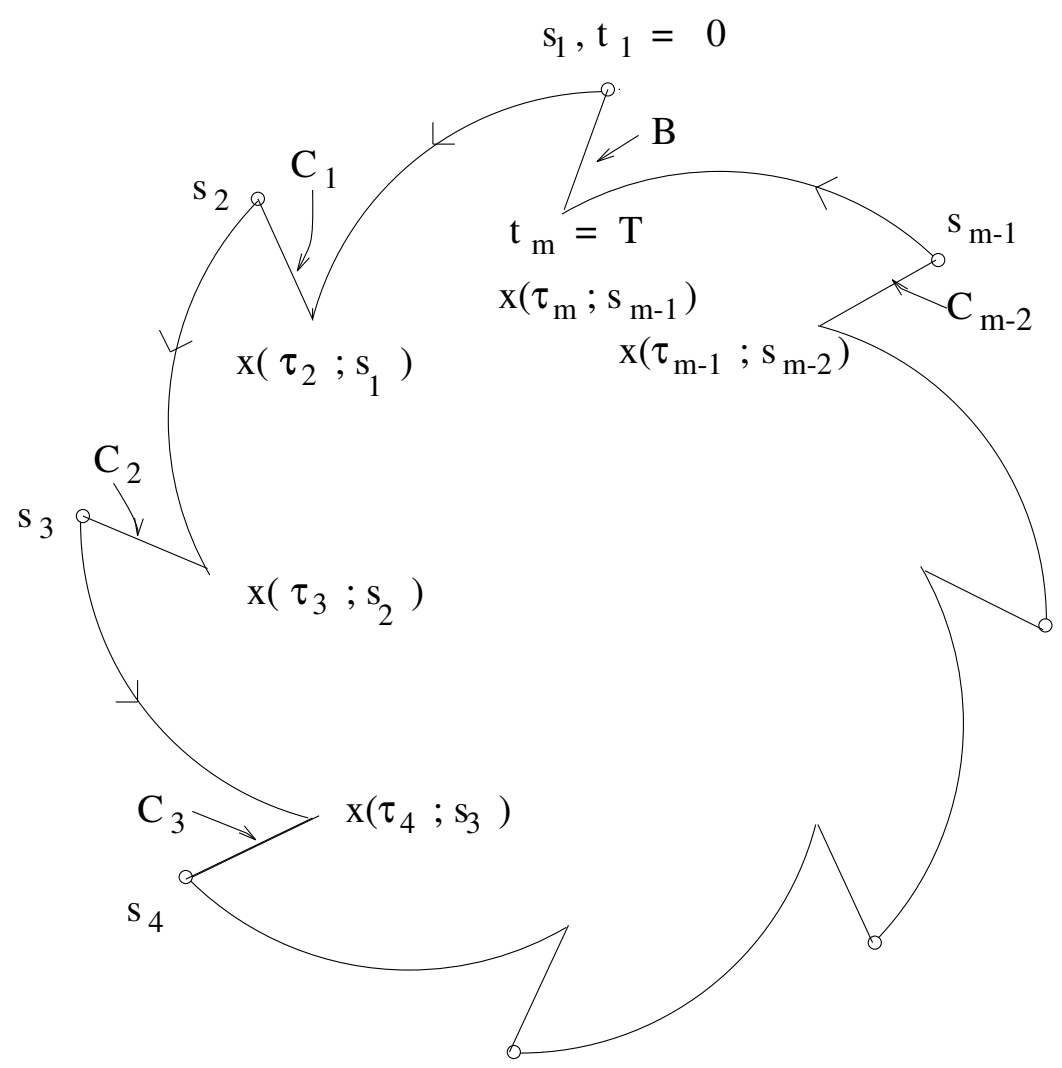

Figure 5: A schematical representation of the multiple shooting procedure applied to periodic orbits.

In this section we drop the index for the iterations $k$, and we use the index $j$ to denote the nodes in time. If the initial conditions of the trajectory at each node $j$ is $\vec{s}_{j}$ at time $\tau_{j}$, and the final 
value of the trajectory at time $\tau_{j+1}$ is denoted by $\vec{x}\left(\tau_{j+1} ; \vec{s}_{j}\right)$, then $(m-2)$ continuity conditions should be satisfied (for a graphical representation see Fig. 5)

$$
\vec{C}_{j}\left(\vec{s}_{j}, \vec{s}_{j+1}\right)=\vec{x}\left(\tau_{j+1} ; \vec{s}_{j}\right)-\vec{s}_{j+1}=0, \quad j=1,2, \cdots, m-2,
$$

together with the boundary conditions

$$
\vec{B}\left(\vec{s}_{1 *}, \vec{s}_{m-1 *}\right)=0 .
$$

Now, we have to solve $(m-1)$ initial value problems, and for that we adopt the Newton-Raphson method

$$
\vec{C}_{j}\left(\vec{s}_{j}+\Delta \vec{s}_{j}, \vec{s}_{j+1}+\Delta \vec{s}_{j+1}\right) \approx \vec{C}_{j}\left(\vec{s}_{j}, \vec{s}_{j+1}\right)+\frac{\partial \vec{C}}{\partial \vec{s}_{j}} \Delta \vec{s}_{j}+\frac{\partial \vec{C}}{\partial \vec{s}_{j+1}} \Delta \vec{s}_{j+1}=0
$$

These equations become

$$
\vec{C}_{j}\left(\vec{s}_{j}, \vec{s}_{j+1}\right)+Z_{j}\left(\tau_{j+1}\right) \Delta \vec{s}_{j}-\Delta \vec{s}_{j+1}=0, \quad 1 \leq j \leq m-2,
$$

where,

$$
Z_{j}\left(\tau_{j+1}\right)=\frac{\partial \vec{x}\left(\tau_{j+1} ; \vec{s}_{j}\right)}{\partial \vec{s}_{j}}
$$

Linearizing the boundary conditions we get

$$
\vec{B}\left(\vec{s}_{1}+\Delta \vec{s}_{1}, \vec{s}_{m-1}+\Delta \vec{s}_{m-1}\right)=\vec{B}\left(\vec{s}_{1}, \vec{s}_{m-1}\right)+\frac{\partial \vec{B}}{\partial \vec{s}_{1}} Z_{1}(0) \Delta \vec{s}_{1}+\frac{\partial \vec{B}}{\partial \vec{s}_{m-1}} Z_{m-1}(1) \Delta \vec{s}_{m-1}=0,
$$

Hence, Eqs $(39,41)$ provide a linear system with $2 N(m-1)$ unknown variables. For periodic orbit boundary conditions these equations in matrix form are written as

$$
\left[\begin{array}{cccccc}
Z_{1} & -I_{2 N} & 0 & \cdots & 0 & 0 \\
0 & Z_{2} & -I_{2 N} & \cdots & 0 & 0 \\
\cdots & \cdots & \cdots & \cdots & \cdots & \cdots \\
0 & 0 & 0 & \cdots & Z_{m-2} & -I_{2 N} \\
-I_{2 N} & 0 & 0 & \cdots & 0 & Z_{m-1}
\end{array}\right]\left[\begin{array}{l}
\Delta \vec{s}_{1} \\
\Delta \vec{s}_{2} \\
\cdots \\
\Delta \vec{s}_{m-2} \\
\Delta \vec{s}_{m-1}
\end{array}\right]=-\left[\begin{array}{l}
\vec{C}_{1} \\
\vec{C}_{2} \\
\cdots \\
\vec{C}_{m-2} \\
\vec{B}
\end{array}\right]
$$

Although each block $Z_{i}$ is not sparse the total matrix is sparse and it can be solved with special routines for distributed computing $[24,25,26]$.

\section{Conclusions}

The algorithms described in the previous sections were used extensively in the past mainly with triatomic molecules $[27,28,29]$. Currently, we are developing the computer codes to apply the classical and quantum algorithms to molecules with biological interest.

\section{Acknowledgment}

Support from the Greek Ministry of Education and European Union through the postgraduate program EPEAEK, "Applied Molecular Spectroscopy", is gratefully acknowledged. LL thanks the Institute of Electronic Structure and Laser-FORTH for the hospitality during his visits to Crete. 


\section{References}

[1] I. Foster and C. Kesselman, (Editors.). The Grid: Blueprint for a new Computing Infrastructure, Morgan Kaufmann (1999).

[2] European Network for Advanced Computing Technology for Science (ENACTS), http://www.epcc.ed.ac.uk/enacts/.

[3] http://tccc.iesl.forth.gr/general/intro/pdf/108.pdf

[4] S. C. Farantos, Exploring Molecular Vibrations with Periodic Orbits, Int. Rev. Phys. Chem. 15, 345-374 (1996).

[5] S. C. Farantos, POMULT: A Program for Computing Periodic Orbits in Hamiltonian Systems Based on Multiple Shooting Algorithms, Comp. Phys. Comm. 108, 240-258 (1998).

[6] R. Kosloff, Quantum Molecular Dynamics on Grids, Dynamics of Molecules and Chemical Reactions, Editors: R. E. Wyatt and J. Z. H. Zhang, Marcel Dekker Inc., N.Y., 185-230 (1996).

[7] R. Kosloff, Propagation Methods for Quantum Molecular Dynamics, Ann. Rev. Phys. Chem. 45, 145-178 (1994).

[8] (a) C. Lanczos, J. Res. Nat. Bur. Stand. 45, 58 (1950); (b) C. Iung and C. Leforestier, Direct calculation of overtones: Application to the $\mathrm{CD}_{3} \mathrm{H}$ molecule, J. Chem. Phys. 102, 8453-8461 (1995); (c) R. E. Wyatt, Computation of high-energy vibrational eigenstates: Application to $\mathrm{C}_{6} \mathrm{H}_{5} \mathrm{D}$, J. Chem. Phys. 103, 8433-8443 (1995); (d) R. B. Lehoucq, D. C. Sorensen and C. Yang, Solution of Large Scale Eigenvalue Problems with Implicitly Restarted Arnoldi Methods, (SIAM, Philadelphia (1998)).

[9] Dynamics of Molecules and Chemical Reactions, Editors: R. E. Wyatt and J. Z. H. Zhang, Marcel Dekker, Inc., N.Y. (1996).

[10] J. Broeckhove and L. Lathouwers, Quantum Dynamics and Angular Momentum Projection, Numerical Grid Methods and their Applications to Schrödinger Equation, Editor: C. Cerjan, Kluwer Academic Publishers, 49-56 (1993).

[11] R. Guantes and S. C. Farantos, High Order Finite Difference Algorithms for Solving the Schrödinger Equation in Molecular Dynamics, J. Chem. Phys. 111, 10827-10835 (1999).

[12] R. Guantes and S. C. Farantos, High Order Finite Difference Algorithms for Solving the Schrödinger Equation in Molecular Dynamics. II. Periodic variables, J. Chem. Phys. 113, 10429-10437 (2000).

[13] R. Guantes, A. Nezis and S. C. Farantos, Periodic Orbit - Quantum Mechanical Investigation of the Inversion Mechanism of $\mathrm{Ar}_{3}$, J. Chem. Phys. 111, 10836-10842 (1999).

[14] (a) B. Fornberg, A Practical Guide to Pseudospectral Methods, Cambridge Monographs on Applied and Computational Mathematics (Cambridge Univ. Press, 1 (1998)); (b) B. Fornberg and D. M. Sloan, Acta Numerica, 203-267 (1994).

[15] J. P. Boyd, A fast algorithm for Chebyshev, Fourier, and sinc interpolation onto an irregular grid, J. Comp. Phys. 103, 243-257 (1992).

[16] J. P. Boyd, Sum-accelerated pseudospectral methods: Finite differences and sech-weighted differences, Comp. Methods Appl. Mech. Engrg. 116, 1-11 (1994). 
[17] D. T. Colbert and W. H. Miller, A novel discrete variable representation for quantum mechanical reactive scattering via the S-matrix Kohn method, J. Chem. Phys. 96, 1982-1991 (1992).

[18] W. H. Press, B. P. Flannery, S. A. Teukolsky and W. T. Vetterling, Numerical Recipes, Cambridge University Press (1986).

[19] (a) J. V. Lill, G. A. Parker and J. C. Light, Discrete variable representations and sudden models in quantum scattering theory, Chem. Phys. Lett. 89, 483-489 (1982); (b) J. C. Light, I. P. Hamilton and J. V. Lill, Generalized discrete variable approximation in quantum mechanics, J. Chem. Phys. 82, 1400-1409 (1985); (c) S. E. Choi and J. C. Light, Determination of the bound and quasibound states of $\mathrm{ArHCl}$ van der Waals complex: Discrete variable representation method, ibid. 92, 2129-2145 (1990).

[20] Brendan Vastenhouw and Rob H. Bisseling, A Two-Dimensional Data Distribution Method for Parallel Sparse Matrix-Vector Multiplication, SIAM Review 47, 67-95 (2005) .

[21] M. Founargiotakis, S. C. Farantos, G. Contopoulos and C. Polymilis, Periodic Orbits, Bifurcations and Quantum Mechanical Eigenfunctions and Spectra, J. Chem.Phys. 91, 1389-1402 (1989).

[22] R. Seydel, From Equilibrium to Chaos: Practical bifurcation and stability analysis, Elsevier (1988).

[23] E. L. Allgower and K. Georg, Numerical Continuation Methods, Springer series in computational mathematics, 13, Berlin:Springer-Verlag (1993).

[24] Satish Balay, Kris Buschelman, William D. Gropp, Dinesh Kaushik, Matthew G. Knepley, Lois Curfman McInnes, Barry F. Smith and Hong Zhang, PETSc, http://www.mcs.anl.gov/petsc (2001).

[25] Satish Balay, Kris Buschelman, Victor Eijkhout, William D. Gropp, Dinesh Kaushik, Matthew G. Knepley, Lois Curfman McInnes, Barry F. Smith and Hong Zhang, PETSc Users Manual, ANL-95/11 - Revision 2.1.5, Argonne National Laboratory (2004).

[26] Satish Balay, Victor Eijkhout, William D. Gropp, Lois Curfman McInnes, Barry F. Smith, Efficient Management of Parallelism in Object Oriented Numerical Software Libraries, in Modern Software Tools in Scientific Computing, Editors: E. Arge, A. M. Bruaset and H. P. Langtangen, 163-202, Birkhäuser Press (1997).

[27] H. Ishikawa, R. W. Field, S. C. Farantos, M. Joyeux, J. Koput, C. Beck and R. Schinke, HCP - CPH Isomerization: Caught in the Act, Annual Review of Physical Chemistry 50, 443-484 (1999).

[28] M. Joyeux, S. C. Farantos and R. Schinke, Highly Excited Motion in Molecules: Saddle-Node Bifurcations and their Fingerprints in Vibrational Spectra, J. Phys. Chem. 106, (feature article) $5407-5421$ (2002).

[29] M. Joyeux, S. Yu. Grebenshchikov, J. Bredenbeck, R. Schinke and S. C. Farantos, Intramolecular Dynamics Along Isomerization and Dissociation Pathways, "Geometrical Structures of Phase Space in Multi-Dimensional Chaos", Editors: I. Prigogine, Stuart Rice, Advances in Chemical Physics 130, 267-303 John Wiley \& Sons,(2005). 